\title{
ON THE PERMANENT AND MAXIMAL CHARACTERISTIC ROOT OF A NONNEGATIVE MATRIX
}

\author{
RICHARD A. BRUALDI ${ }^{1}$
}

One of the many theorems that M. Marcus and M. Newman prove in [3] is, when it is restricted to nonnegative matrices, that: if $A$ is an $n \times n$ positive semidefinite symmetric irreducible nonnegative matrix, then

$$
\lim _{m \rightarrow \infty}\left(\operatorname{per}\left(A^{m}\right)\right)^{1 / m}=r^{n}
$$

where $r$ denotes the maximal (positive) characteristic root of $A$. Here per $\left(A^{m}\right)$ denotes the permanent of $A^{m}$. We assume that the reader is familiar with the terminology and results of the classical PerronFrobenius-Wielandt theory of nonnegative matrices, the requisite parts of which can be found in [1, Chapter XIII].

The purpose of this note is to prove the following extension of the result quoted above.

Theorem. Let $A$ be an $n \times n$ nonnegative irreducible matrix and suppose $A$ has a nonzero permanent. Let $r$ be the maximal characteristic root of $A$. Then

$$
\lim _{m \rightarrow \infty}\left(\operatorname{per}\left(A^{m}\right)\right)^{1 / m}=r^{n} .
$$

We first prove two lemmas.

LeMma 1. Let $B$ be an $n \times n$ nonnegative matrix with maximal characteristic root $\rho$. Then

$$
\operatorname{per}(B) \leqq \rho^{n} \text {. }
$$

Proof. ${ }^{2}$ First assume $A$ is positive (or irreducible). Then $\rho$ is positive and has an associated characteristic vector $x=\left(x_{1}, \cdots, x_{n}\right)$, all of whose coordinates are positive. From $B x=\rho x$, it follows that

$$
\sum_{j=1}^{n} b_{i j} x_{j}=\rho x_{i}, \quad i=1, \cdots, n
$$

and hence

Received by the editors March 18, 1966.

1 The research of the author was partially supported by NSF grant no. GP-3993.

2 This is a slight variation of a proof originally shown the author by Morris Newman. 


$$
\prod_{i=1}^{n}\left(\sum_{j=1}^{n} b_{i j} x_{j}\right)=\rho^{n} \prod_{i=1}^{n} x_{i}
$$

This may be written as

$$
\operatorname{per}(B) \prod_{i=1}^{n} x_{i}+\beta=\rho^{n} \prod_{i=1}^{n} x_{i}
$$

where $\beta \geqq 0$ and the result follows. The complete lemma now follows by continuity.

LEMмa 2. Let $A$ be an $n \times n$ nonnegative primitive matrix with maximal characteristic root $r$. Then (1) holds.

Proof. If $m$ is a positive integer, then using Lemma 1 and the fact that $A^{m}$ is nonnegative, we may write

$$
\prod_{i=1}^{n} a_{i i}^{(m)} \leqq \operatorname{per}\left(A^{m}\right) \leqq\left(r^{m}\right)^{n}
$$

where $a_{i j}^{(m)}$ denotes the $(i, j)$ entry of $A^{m}$. Thus we have

$$
\prod_{i=1}^{n}\left(a_{i i}^{(m)}\right)^{1 / m} \leqq \operatorname{per}\left(A^{m}\right)^{1 / m} \leqq r^{n} .
$$

But it is well known (see [2, p. 128] or $[1$, p. 81]) that for a primitive matrix $A$,

$$
\lim _{m \rightarrow \infty}\left(a_{i j}^{(m)}\right)^{1 / m}=r .
$$

Taking limits in (2), we obtain (1).

Proof of Theorem. Let $h$ denote the index of imprimitivity of $A$, that is, the number of characteristic roots of $A$ of modulus $r$. Then $[1$, p. 81-82] there is a permutation matrix $P$ such that

$$
P^{T} A^{h} P=\operatorname{diag}\left(A_{1}, A_{2}, \cdots, A_{h}\right)
$$

where each $A_{i}, 1 \leqq i \leqq h$, is an $n_{i} \times n_{i}$ primitive matrix with maximal characteristic root $r^{h}$. Observe that if $k$ is a positive integer, then

$$
\left(P^{T} A^{h} P\right)^{k}=P^{T} A^{h k} P=\operatorname{diag}\left(A_{1}^{k}, A_{2}^{k}, \cdots, A_{h}^{k}\right) .
$$

Since the permanent of a matrix is invariant under permutations, we have

$$
\left(\operatorname{per}\left(A^{h k}\right)\right)^{1 / h k}=\left[\left(\operatorname{per}\left(A_{1}^{k}\right)\right)^{1 / k} \cdots\left(\operatorname{per}\left(A_{h}^{k}\right)\right)^{1 / k}\right]^{1 / h}
$$


Since each $A_{i}$ is a primitive matrix, it follows by Lemma 2, that

$$
\lim _{k \rightarrow \infty}\left(\operatorname{per}\left(A^{h k}\right)\right)^{1 / h k}=\left(\prod_{i=1}^{h}\left(r^{h}\right)^{n_{i}}\right)^{1 / h}=r^{n} .
$$

Now let $m$ be an arbitrary positive integer and write

$$
m=h p_{m}+q_{m} ; \quad p_{m} \geqq 0, \quad 0 \leqq q_{m}<h .
$$

Then

$$
A^{m}=A^{h p_{m}} A^{q_{m}}
$$

where $A^{q_{m}}=I$ if $q_{m}=0$. Thus since all matrices are nonnegative

$$
\operatorname{per}\left(A^{m}\right) \geqq \operatorname{per}\left(A^{h p_{m}}\right) \operatorname{per}\left(A^{q_{m}}\right) \text {. }
$$

From (3) it follows immediately that

$$
\lim _{m \rightarrow \infty}\left(\operatorname{per}\left(A^{h p_{m}}\right)\right)^{1 / h p_{m}}=r^{n} .
$$

But then using (4) we obtain

$$
\begin{gathered}
\lim _{m \rightarrow \infty}\left(\ln \left(\operatorname{per}\left(A^{h p_{m}}\right)\right)^{1 / h p_{m}}-\ln \left(\operatorname{per}\left(A^{h p_{m}}\right)^{1 / m}\right)\right) \\
=\lim _{m \rightarrow \infty} \frac{q_{m}}{m} \ln \left(\operatorname{per}\left(A^{h p_{m}}\right)\right)^{1 / h p_{m}}=0 .
\end{gathered}
$$

Hence

$$
\lim _{m \rightarrow \infty} \frac{\left(\operatorname{per}\left(A^{h p_{m}}\right)\right)^{1 / h p_{m}}}{\left(\operatorname{per}\left(A^{h p_{m}}\right)\right)^{1 / m}}=1 .
$$

Now (4) and (5) together imply

$$
\lim _{m \rightarrow \infty}\left(\operatorname{per}\left(A^{h p_{m}}\right)\right)^{1 / m}=r^{n} .
$$

Now by assumption per $(A)>0$. If per $(A) \geqq 1$, then

$$
\text { per }\left(A^{q_{m}}\right) \geqq(\operatorname{per}(A))^{q_{m}} \geqq 1, \quad \text { for all } m \text {; }
$$

while if per $(A)<1$, then

$$
\operatorname{per}\left(A^{q_{m}}\right) \geqq(\operatorname{per}(A))^{q_{m}} \geqq \operatorname{per}(A)^{h-1}, \quad \text { for all } m \text {. }
$$

If $r \geqq 1$, then by Lemma 1

$$
\text { per }\left(A^{q_{m}}\right) \leqq\left(r^{n}\right)^{q_{m}} \leqq r^{n(h-1)}, \quad \text { for all } m ;
$$

while if $r \leqq 1$, then 


$$
\operatorname{per}\left(A^{q_{m}}\right) \leqq\left(r^{n}\right)^{q_{m}} \leqq 1, \quad \text { for all } m .
$$

Thus in any case there are positive constants $c_{1}$ and $c_{2}$ such that

$$
c_{1} \leqq \operatorname{per}\left(A^{q_{m}}\right) \leqq c_{2}
$$

for all positive integers $m$. From this it now follows that

$$
\lim _{m \rightarrow \infty}\left(\operatorname{per}\left(A^{q_{m}}\right)\right)^{1 / m}=1 .
$$

Passing to the limit in the inequality

$$
\left(\operatorname{per}\left(A^{h p_{m}}\right)\right)^{1 / m}\left(\operatorname{per}\left(A^{q_{m}}\right)\right)^{1 / m} \leqq\left(\operatorname{per}\left(A^{m}\right)\right)^{1 / m} \leqq r^{n},
$$

and using (6) and (7), we obtain the desired result.

The condition that $A$ have a nonzero permanent cannot in general be omitted. The irreducible matrix

$$
A=\left[\begin{array}{lll}
0 & 1 & 0 \\
1 & 0 & 1 \\
0 & 1 & 0
\end{array}\right]
$$

has the property that per $\left(A^{k}\right)=0$ for $k$ odd and per $\left(A^{k}\right)>0$ for $k$ even. It is easy to verify that $A$ has maximal characteristic root $2^{1 / 2}$ and index of imprimitivity 2. It follows from (3) that if $\lim _{m \rightarrow \infty}\left(\operatorname{per}\left(A^{m}\right)\right)^{1 / n}$ exists, it must be $2^{3 / 2}$. Hence the limit does not even exist.

Finally we remark that our theorem does constitute an extension of the result of Marcus and Newman mentioned in the beginning. For, if $A$ is a positive semidefinite symmetric irreducible nonnegative matrix, then the entries on the main diagonal of $A$ must be positive. Otherwise $A$ has a zero row and thus is not irreducible. Hence the permanent of such a matrix is positive.

\section{REFERENCES}

1. F. R. Gantmacher, The theory of matrices, Vol. II, Chelsea, New York, 1959.

2. M. Marcus and H. Minc, A survey of matrix theory and matrix inequalities, Allyn and Bacon, Boston, 1964.

3. M. Marcus and M. Newman, Inequalities for the permanent function, Ann. of Math. (2) 75 (1962), 47-62.

UNiversity OF Wisconsin 